\title{
Occurrence and Breaking of Rogue Waves in Deep Waters: A Stochastic Approach Revisit
}

\author{
Ioannis Alex Papadimitrakis ${ }^{1, *}$ and Frédéric Dias ${ }^{2,3}$

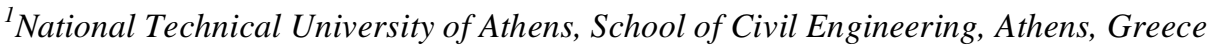 \\ ${ }^{2}$ Ecole Normale Supérieure de Cachan, CMLA, Cachan, France \\ ${ }^{3}$ University College Dublin, School of Mathematical Sciences, Dublin, Ireland
}

\begin{abstract}
The occurrence of rogue waves in deep sea waters, and their breaking, is examined with the aid of a pdf model of joint amplitudes and frequencies. New wave breaking considerations allow kinematic, dynamic and maximum average slope concepts to be unified in a single breaking criterion, which allows a more accurate determination of the limiting amplitudes that rogue waves can reach, including the influence of non-linearity of the wave field and the premature wave breaking concept. The probability of rogue wave occurrence does not significantly depend on the sea spectrum bandwidth. The breaking probability of rogue waves increases with the inverse wave age, but its dependence on the latter parameter weakens, as the limiting crest height criterion is stiffened. The right variation of sea surface kurtosis, and of the Benjamin Feir Index, with the (inverse) wave age and their lower than unity values reconfirms their relation to the rogue wave occurrence.
\end{abstract}

Key Words: Rogue waves, stochastic breaking, breaking criteria, BFI, sea surface kurtosis.

\section{INTRODUCTION}

The presence of rogue waves, and in particular their breaking, threatens offshore structures, ships and wave energy converters, and may cause a lot of damage to them, possibly their destruction, and the loss of human lives. Quantitative information on the breaking of surface waves, in deep water, is important in many aspects of oceanography, ocean engineering, marine hydrodynamics and environmental marine engineering, since wave breaking is the primary mechanism of wave energy dissipation and the major source of turbulence in the surface marine layer; it also enhances the exchange of gas, water vapor, momentum and energy between the atmosphere and the ocean. The interested reader may find valuable information on this subject (of wave breaking) in the recent book of Massel [1]. Large scale breaking may also cause the splitting of large oil slicks into smaller pieces, some of which enter the water column with the aid of the plunging crest tips. There, these smaller parcels either form water-in oil emulsions or dissolve in the water, forming oil-in water emulsions, or possibly adhere to other particles in suspension, causing in all cases great damage to the marine ecosystem.

Conventionally, rogue waves are surface gravity waves whose heights are much larger than expected for a given sea state (see precise definitions below). Such a picture is clearly seen in time records of sea surface height, during many storms, as for example in records from the Gorm and Draupner storms ([2]; the reader interested in this subject should peruse this quite informative reference). Rogue waves are recognized when $\mathrm{H}_{\mathrm{h}} / \mathrm{H}_{\mathrm{s}}>2$ or $\mathrm{H}_{\mathrm{c}} / \mathrm{H}_{\mathrm{s}}>1.25$, although other

*Address correspondence to this author at the National Technical University of Athens, School of Civil Engineering, Athens, Greece; Tel: +302107722876; Fax: +30-2107722814; E-mail: ypapadim@central.ntua.gr authors have adopted values as low as 1.1, for the second inequality; here $\mathrm{H}_{\mathrm{h}}, \mathrm{H}_{\mathrm{s}}, \mathrm{H}_{\mathrm{c}}$ are the wave height, the significant wave height and the crest wave height (or amplitude), respectively. The occurrence of rogue waves is also found, in the literature, to relate to other parameters that express either the sea surface characteristics or some of its properties, such as the sea wave spectral bandwidth $\theta$, a measure of the sea surface slope (like the rms slope, $S_{\mathrm{rms}}$, or the significant slope, $\S$ ), the sea surface kurtosis $\mathrm{C}_{4}$, and the Benjamin Feir Index, BFI; $\mathrm{C}_{4}$ appears to be related to the BFI [3, 4]. Definitions of these quantities follow.

Rogue waves are formed by different mechanisms that have been described by various authors (see, for example, the excellent review [2]), namely: a) non-linear wave energy focusing caused by currents interacting with surface gravity waves, generated locally or elsewhere and propagating either as swell or another sea, forming a mixed sea, b) dispersive spatial-temporal focusing, i.e., by constructive interference of dispersive wave components in space and/or time (as in wave-wave interactions), c) topographic focusing in shallow coastal waters, via bottom diffraction and/or variable current refraction and by coastal or caustic reflection, and d) nonlinear instability mechanisms, such as the Benjamin-Feir mechanism. In summary, rogue waves may be generated by meteorological, dispersive and/or topographic focusing, wave-current and wave-wave interactions. The generation of rogue waves can also be experienced in laboratory experiments, where waves propagate along co-flowing and/or opposing currents [5].

The objectives of this study are: i) the prediction of occurrence of rogue waves and their breaking, under various sea state conditions (expressed by the slope, $\S$, and/or the wave age, $c_{\mathrm{p}} / \mathrm{U}_{10}$ ), using a stochastic model of the joint $\mathrm{pdf}$ 
of amplitudes and frequencies of sea waves described by Papadimitrakis ([6], called hereafter IP), and ii) the exploration of a possible relation of rogue wave occurrence with other established indices, such as $\theta, \mathrm{S}_{\mathrm{rms}}$ or $\S, \mathrm{C}_{4}$ and $\mathrm{BFI}$, using the above joint pdf model. Here, $c_{p}$ is the phase speed of the dominant wave (i.e., of the wave component having the spectral peak frequency) and $U_{10}$ is the wind speed, measured at a height of $10 \mathrm{~m}$ above the mean sea level (MSL).

\section{THE STOCHASTIC MODEL}

The joint distribution of amplitudes and frequencies, $\mathrm{p}(\mathrm{h}$, $\omega)$, has been described in detail by IP. This model applies to both narrow- and broadband seas. Its spectral density, in terms of normalized amplitude, $\mathrm{H}$, and frequency, $\Sigma$, is given by:

$$
\begin{aligned}
& p(\mathrm{H}, \Sigma)=8 \mathrm{H}^{2} \Sigma^{3} /\left\{\pi^{1 / 2}\left(\theta^{2}-1\right)^{1 / 2}(\theta+1)\right\} \\
& \exp \left\{-H^{2}\left[1+\left(\Sigma^{2}-1\right)^{2} /\left(\theta^{2}-1\right)\right]\right\} \\
& H=h /\left(2 m_{0}\right)^{1 / 2}, \quad \Sigma=\omega /\left(\alpha^{1 / 2} \omega_{0}\right), \\
& \omega_{0}=\left(m_{2} / m_{0}\right)^{1 / 2}, \quad \theta=\left(m_{0} m_{4} / m_{2}^{2}\right)^{1 / 2} \\
& \Sigma_{\mathrm{p}}\left(=\alpha^{-1 / 2} \omega_{\mathrm{p}} / \omega_{0}\right)=\left\{\left[2+\left(4+21 \theta^{2}\right)^{1 / 2}\right] / 7\right\}^{1 / 2} \\
& (1 \mathrm{a}, \mathrm{b}, \mathrm{c}, \mathrm{d}, \mathrm{e}, \mathrm{f}, \mathrm{g})
\end{aligned}
$$

where $\mathrm{h}, \omega$ are the amplitude and radian frequency, $\mathrm{m}_{\mathrm{i}}(\mathrm{i}=0$, $1,2,4)$ is the ith moment of the sea spectrum, and $\alpha$ is a positive coefficient, dependent also on $\theta, \omega_{\mathrm{o}}$ and $\omega_{\mathrm{p}}$; the subscript $\mathrm{p}$ refers to the spectral peak. Upon integration of $\mathrm{p}(\mathrm{H}$, $\Sigma$ ) with respect to either $\Sigma$ or $\mathrm{H}$, the amplitude and frequency marginal densities are obtained, namely:

$$
\begin{aligned}
& p(\mathrm{H})=[2 /(\theta+1)] F(\mathrm{~B}) \mathrm{H} \exp \left(-\mathrm{H}^{2}\right), \\
& \mathrm{p}(\Sigma)=2(\theta-1)\left\{\Sigma /\left[\theta^{2}-1+\left(\Sigma^{2}-1\right)^{2}\right]^{1 / 2}\right\}^{3} \\
& F(\mathrm{~B})=1+\operatorname{erf}\left(\mathrm{B}^{-1}\right)+\left(\mathrm{B} \pi^{-1 / 2}\right) \exp \left(-\mathrm{B}^{-2}\right), \\
& \mathrm{B}=\left(\theta^{2}-1\right)^{1 / 2} / \mathrm{H}
\end{aligned}
$$$$
(2 \mathrm{a}, \mathrm{b}, \mathrm{c}, \mathrm{d})
$$

The first moment of $\mathrm{p}(\mathrm{H})$ distribution provides the average amplitude, $\mathrm{H}_{\mathrm{av}}$, an exclusive function of bandwidth, $\theta$, that also characterizes a given sea state. Detailed expressions of $\mathrm{p}(\mathrm{H}), \mathrm{p}(\Sigma)$ and $\mathrm{H}_{\mathrm{av}}$ can be found in IP. Two more quantities of interest may be obtained from $\mathrm{p}(\mathrm{H}, \Sigma)$ upon division of the latter by $\mathrm{p}(\mathrm{H})$ or $\mathrm{p}(\Sigma)$, respectively, namely the conditional frequency and/or amplitude probabilities, given as:

$$
\begin{aligned}
& \mathrm{p}(\Sigma \mid \mathrm{H})=p(H, \Sigma) / p(H)=\left\{4 \mathrm{H} \Sigma^{3} /\left[\pi^{1 / 2}\left(\theta^{2}-1\right)^{1 / 2}\right]\right\} . \\
& \exp \left[-\mathrm{H}^{2}\left(1-\Sigma^{2}\right)^{2} /\left(\theta^{2}-1\right)\right] \cdot \mathrm{F}^{-1}\left(\mathrm{~B}_{\mathrm{m}}\right) \\
& \mathrm{p}(\mathrm{H} \mid \Sigma)=p(H, \Sigma) / p(\Sigma) \quad(3 \mathrm{a}, \mathrm{b}, \mathrm{c})
\end{aligned}
$$

The $\mathrm{p}(\mathrm{H}), \mathrm{p}(\mathrm{H} \mid \Sigma)$ and/or $\mathrm{p}(\Sigma \mid \mathrm{H})$ distributions are particularly important in exploring both the occurrence and the breaking of rogue waves.

\section{WAVE BREAKING CONSIDERATIONS}

Waves in the ocean travel in groups [7-10]. The association of breaking waves, at sea, with wave group structures has been reported a long time ago by Donelan et al. [11]. Assuming that the length of a rogue wave is comparable to the dominant wave length $\lambda_{\mathrm{p}}$, it follows that rogue waves are relatively steep. In a group of waves, when the wave at the peak of the group envelope becomes sufficiently steep, exceeding a threshold of critical steepness $(\mathrm{ak})_{\mathrm{cr}}$, it will break. Thus, wave breaking (of the dominant wave) and rogue wave presence are intimately related [12].

In order to improve the understanding of wave breaking processes, it is necessary to focus on the proper criteria characterizing the occurrence of wave breaking, considering the accumulated knowledge from field and laboratory observations on this aspect. Papadimitrakis [6] has described how the breaking criteria of Phillips [13] can be used, in conjunction with his joint $\mathrm{p}(\mathrm{H}, \Sigma) \mathrm{pdf}$, to generate the analytical form of spectral wave breaking probability, $\mathrm{p}_{\mathrm{B}}(\Sigma)$, at any frequency $\Sigma$. These criteria describe the limiting amplitude, $\mathrm{h}_{\mathrm{o}}$, that a wave of frequency, $\omega$, can reach (in the absence or presence of swell) before it breaks, but they have been modified (by IP) to properly account for surface drift current and non-linearity effects of the wave field. The latter are expressed in a rather implicit way through the function $f$ (see pg. 4) and not by properly transforming the joint pdf, $\mathrm{p}(\mathrm{H}$, $\Sigma$ ), to directly incorporate the second and/or higher order effects of wave non-linearities into the expressions that provide both the pdf of the crest amplitude (or crest height) and the breaking pdf, $\mathrm{p}_{\mathrm{B}}(\Sigma)$, as done by other authors for deep and/or shallow waters (e.g. [14-18]). Such and other aspects, as, for example, how the irregularity and unsteadiness of water waves affect their breaking process and how the shortcrest (3D) and long-crest (2D) wave features (or other important characteristics, as wave directionality, etc.) influence the occurrence and breaking (in particular) of rogue waves, will be considered in detail in a forthcoming paper. It is worth noting that, in the open ocean (i.e. in deep water), second order non-linearities may not have a significant impact on the above quantities of interest, examined in this study, as they do have for shallow waters [19], but this remains to be seen. 
Of particular interest, in this study, is the variation of $\mathrm{p}_{\mathrm{B}}\left(\Sigma_{\mathrm{p}}\right)$, i.e., of the breaking probability of the dominant wave as a function of an overall wave steepness, a parameter that characterizes locally the entire wave spectrum. Such steepness may be represented by:

$$
\S\left[=m_{0}^{1 / 2} \omega_{p}^{2} /(2 \pi g)\right] \text { or } \mathrm{S}_{\mathrm{rms}}\left[=g^{-2} \int_{0}^{\infty} \omega^{4} S(\omega) d \omega\right]^{1 / 2}
$$

Here, $g$ is the acceleration due to gravity and $S(\omega)$ represents the 1D wave spectrum. It might be argued that $\mathrm{S}_{\mathrm{rms}}=\mathrm{c}_{2} \S$, where the numerical coefficient $c_{2}$ is of $\mathrm{O}(2 \pi)$; its actual value depends on the form of the spectrum used to estimate $\mathrm{S}_{\mathrm{rms}}$.

As described in IP, $\mathrm{p}_{\mathrm{B}}\left(\Sigma_{\mathrm{p}}\right)$ appears to increase dramatically with $\S$; furthermore, at frequencies $2 \Sigma_{\mathrm{p}}$ and $2.5 \Sigma_{\mathrm{p}}$ the corresponding $\mathrm{p}_{\mathrm{B}}\left(2 \Sigma_{\mathrm{p}}\right)$ and $\mathrm{p}_{\mathrm{B}}\left(2.5 \Sigma_{\mathrm{p}}\right)$ distributions lie below the $\mathrm{p}_{\mathrm{B}}\left(\Sigma_{\mathrm{p}}\right)$ curve and vary slowly with $\mathrm{c}_{\mathrm{p}} / \mathrm{u}_{*}$ ( $\mathrm{u}_{*}$ being the wind friction velocity), in agreement with the Banner et al. results $[20,21]$. The latter authors have also explored the behavior of $\mathrm{p}_{\mathrm{B}}\left(\omega_{\mathrm{p}}\right)$ distribution with the saturation function $\sigma_{f}(\omega)$, which in terms of non-dimensional frequency $\Sigma$ is given by:

$\sigma_{f}(\Sigma)\left[=\left(2 g^{2}\right)^{-1}\left(\Sigma \alpha^{1 / 2} \omega_{o}\right)^{5} S\left(\Sigma \alpha^{1 / 2} \omega_{o}\right)\right]$

Banner et al. $[20,21]$ found that $\mathrm{p}_{\mathrm{B}}\left(\omega_{\mathrm{p}}\right)$ increases rather linearly in various $\sigma_{f}(\omega)$ regions, slower in the lower $\sigma_{f}(\omega)$ value region and more abruptly in the higher $\sigma_{f}(\omega)$ value region. The behavior of $\mathrm{p}_{\mathrm{B}}\left(\Sigma_{\mathrm{p}}\right)$ distribution with $\sigma_{f}(\Sigma)$ is also explored in this study.

\section{3a. Local maximum and Average Wave Slope Aspects}

Combining the local limiting amplitude, $\mathrm{h}_{\mathrm{o}}$, with the corresponding wave number, it is possible to derive an expression for the local maximum slope, $S_{\max }(\Sigma)$, at the corresponding frequency, $\Sigma$. As described in IP, these local slopes are reduced considerably, by the influence of surface drift, at frequencies remote from the spectral peak. Neglecting drift current effects, near the spectral peak, the maximum slope at $\Sigma_{\mathrm{p}}$ may be written as:

$\mathrm{S}_{\max , \mathrm{p}}=\mathrm{S}_{\max }\left(\Sigma_{\mathrm{p}}\right) \cong f /\left(2 \alpha_{1}\right)$

here $f$ is a function that accounts for the non-linearity of the wave field, and $\alpha_{1}$ is a constant that expresses the fact that a wave breaks (at the crest) when the real downward Lagrangian acceleration, $a_{L}$, reaches a value close to $0.39 \mathrm{~g}$ (not $0.5 \mathrm{~g}$ ). Various expressions for $f$ have been provided by Longuet-Higgins [22] and Longuet-Higgins and Fox [23], and are summarized in IP. For $\S \leq \S_{\max }=0.0353, S_{\max , p}$ remains $\leq$ 0.4432. Since $S_{\max , \mathrm{p}} \cong f /\left(2 \alpha_{1}\right)$, this maximum slope appears to increase, with increasing $\S$, from about 0.235 to 0.443 (when $\alpha_{1} \approx 1.39$ ), consistent with field obser- vations. It is noted that both Müller et al. ([24], cf. their Fig. 9) and Donelan and Magnusson [25] have used as a critical steepness (ak) cr for breaking (of rogue) waves (at the crest of a group envelope) the value of 0.3. Low overall steepness $\S$ may also reduce the maximum real acceleration, $a_{L}$, that an individual wave crest can reach, before it breaks, and thus lowers the local limiting slope, $S_{\max }(\Sigma)$, at a particular frequency.

The breaking probability, $\mathrm{p}_{\mathrm{B}}\left(\Sigma_{\mathrm{p}}\right)$, as a function of an overall wave steepness (say $\S$ ), may also be obtained in terms of the local slope at the spectral peak frequency, $S_{p}=$ $\mathrm{S}\left(\Sigma_{\mathrm{p}}\right)$, by estimating the probability of exceedance, $p\left(S_{p}>S_{\max , p}\right)$, of the local maximum slope there, $\mathrm{S}_{\max , \mathrm{p}}=\mathrm{S}_{\max }\left(\Sigma_{\mathrm{p}}\right)$, namely:

$$
p\left(S_{p}>S_{\max , p}\right)=\int_{S_{\max , p}}^{\infty} p\left(S_{p}\right) d S_{p}
$$

In order to accomplish that, it is necessary to estimate first the local maximum slopes, $S_{\max }(\Sigma)$. These slopes, near the spectral peak (where the influence of surface drift is rather small), are given by:

$$
S(\Sigma)=2 \sqrt{2} \pi \S \Sigma_{\mathrm{p}}^{-2}\left(\Sigma^{2} H\right)
$$

Therefore, at the spectral peak: $\mathrm{S}_{\mathrm{p}}=\mathrm{S}\left(\Sigma_{\mathrm{p}}\right)=2 \sqrt{2} \pi \S H=$ $\mathrm{c}_{1} \mathrm{H}$, where $\mathrm{c}_{1}=2 \sqrt{2} \pi \S$. Hence, it follows that $\mathrm{S}_{\max , \mathrm{p}}=\mathrm{S}$ $\max \left(\Sigma_{\mathrm{p}}\right)=2 \sqrt{2} \pi \S H_{o, p}=\mathrm{c}_{1} \mathrm{H}_{\mathrm{o}, \mathrm{p}}$, where $\mathrm{H}_{\mathrm{o}, \mathrm{p}}$ is the limiting normalized crest height at $\Sigma_{\mathrm{p}}$. Following the rules of probability transformation, it is found that:

$$
\begin{aligned}
& p\left(S_{p}\right)=\left.c_{1}^{-1} p(H)\right|_{H=c_{1}^{-1} S_{p}}, p\left(S_{\max , p}\right)=\left.c_{1}^{-1} p(H)\right|_{H=c_{1}^{-1} S_{\max , p},}, \\
& p\left(c_{1} S_{p}\right)=\left.p(H)\right|_{H=c_{1}^{-1} S_{p}}, p\left(S_{p}\right)= \\
& c_{1}^{-1}\left[\mathrm{~F}\left(\mathrm{~B}_{\mathrm{m}}\right) /(\theta+1)\right] 2\left(c_{1}^{-1} \mathrm{~S}_{\mathrm{p}}\right) \exp \left(-c_{1}^{-2} S_{p}^{2}\right), \\
& \mathrm{B}_{\mathrm{m}}=\left(\mathrm{S}_{\mathrm{p}} / c_{1}\right)\left(\theta^{2}-1\right)^{1 / 2}
\end{aligned}
$$

The average slope, $(\mathrm{kh})_{\mathrm{av}}$, characterizing a wave field is obtained now by combining the average amplitude, $\mathrm{h}_{\mathrm{av}}$, and the wave number at the spectral peak frequency, $\mathrm{k}_{\mathrm{p}}$. In terms of $\S,(\mathrm{kh})_{\mathrm{av}}$ is written as:

$$
(\mathrm{kh})_{\mathrm{av}}=2 \sqrt{2} \pi \S \mathrm{H}_{\mathrm{av}}=8.886 \S \mathrm{H}_{\mathrm{av}}
$$

The average slope plays an important role in premature breaking, a concept that has also been described in IP. This premature breaking attribute, and its contribution in forming new unified breaking criteria, are further explored and utilized in this study, as described in section 5. 


\section{3b. Rogue Wave Occurrence and Wave Breaking Relation}

The common physical framework that rogue wave formation and the onset of wave breaking share is the energy focusing within non-linear wave groups. Frequently, a question arises as to what differentiates (large scale) wave breaking from rogue waves. It appears that unidirectionality and directionality effects provide the link and/or the separating boundary between the two sides of the same generic mechanism, the non-linear (wave-wave) interactions within wave group structures. Other related questions that arise are: what are the occurrence rates of such steep waves (rogue waves) and when do they break, whether the statistical joint distribution model of IP can be used to obtain the probability of occurrence of rogue waves, and whether the latter probability is less than $\mathrm{p}_{\mathrm{B}}\left(\Sigma_{\mathrm{p}}\right)$, as indicated from field observations [20]. Answers to these questions may be provided with the aid of $\mathrm{p}(\mathrm{H}), \mathrm{p}(\Sigma \mid \mathrm{H})$ or $\mathrm{p}(\mathrm{H} \mid \Sigma)$ distributions, given previously. More specifically, taking into account the rogue wave definitions, in terms of either $\mathrm{H}_{h}$ or $\mathrm{H}_{c}$, it is possible to evaluate the quantities:

a) $p_{B}\left(\Sigma_{p}\right)$,

b) $\mathrm{p}\left(\mathrm{H}>1.25 \mathrm{H}_{\mathrm{s}}^{\mathrm{n}}\right)$,

c) $p_{\text {orw }}\left(\Sigma_{p}\right)=\left.\int_{H_{c r}}^{\infty} p(H \mid \Sigma) d H\right|_{\Sigma=\Sigma_{p}}$,

d) $B_{o r w, a v}=\int_{H_{c r}}^{\infty} \int_{0}^{\infty} p(H \mid \Sigma) d \Sigma d H$ and

e) $p\left(H>H_{c r, b}\right)=\int_{H_{c r, b}}^{\infty} p(H) d H$

$(11 \mathrm{a}, \mathrm{b}, \mathrm{c}, \mathrm{d}, \mathrm{e})$

Here, $\mathrm{H}_{\mathrm{cr}}=\mathrm{n} H_{s}^{n}, H_{s}^{n}(=2 \sqrt{2})$ is the normalized significant wave height (using our definition of normalization), and $\mathrm{n}=$ $0.0,0.1,0.2, . ., 3.0$, Definitions of $\mathrm{H}_{\mathrm{cr}, \mathrm{b}}$ are given in section 4 .

\section{BREAKING OF ROGUE WAVES}

As described before, breaking of the spectral peak occurs when the local maximum slope $S_{\mathrm{p}}>S_{\max , \mathrm{p}}\left\{\cong f /\left(2 \alpha_{1}\right\}\right.$, or alternatively when the limiting amplitude there $\mathrm{H}_{\mathrm{o}, \mathrm{p}}$ exceeds the threshold value:

$H_{c r, b}^{i p}\left(\cong\left(4 \sqrt{2} \pi \alpha_{1} \S\right)^{-1} \cdot f\right.$

(see also IP). Combining the arguments of Donelan and Magnusson [25] and the results of IP expressing $S_{\max , p}$ as above, and taking into account that the breaking rogue wave slope limit of 0.3 is well within the limits of $S_{\text {max,p }}$ variation (between 0.235 and 0.443 ), it follows that:

$$
\pi H_{o, h} / \lambda_{p} \geq f /\left(2 \alpha_{1}\right), \pi H_{s} / \lambda_{p}=0.1\left(c_{p} / U_{10}\right)^{0.35}
$$

here $H_{o, h}$ represents the height of a breaking rogue wave. Thus:

$$
H_{o, h} / H_{s} \geq 10\left[f /\left(2 \alpha_{1}\right)\right]\left(c_{p} / U_{10}\right)^{0.35}
$$

It is interesting to note that the application of the latter expression, with $c_{p} / U_{10}=1.2$ or 0.3 (or less), yields $H_{o, h} / H_{s}>4.5$ and 1.7 , respectively. This implies that for mature wave fields, the wave height for breaking, $\mathrm{H}_{\mathrm{o}, \mathrm{h}}$, must become very large (i.e. $>4.5 \mathrm{H}_{\mathrm{s}}$ ) compared to that required for less mature or young (and rather choppy, 3D) waves for which $\mathrm{H}_{\mathrm{o}, \mathrm{h}}$ needs to only exceed $1.7 \mathrm{H}_{\mathrm{s}}$, a very sensible result. In terms of the significant slope, it appears that $\S$ must exceed the values 0.03 and 0.074 , respectively when:

$$
\S \cong 3.31 \times 10^{-2}\left(c_{p} / U_{10}\right)^{-0.35}
$$

Using the normalization suggested by IP, for both $\mathrm{H}_{\mathrm{o}, \mathrm{h}}$ and $\mathrm{H}_{\mathrm{s}}$, the non-dimensional limiting crest amplitude is written as:

$$
H_{c r, b}^{d m}=10 \sqrt{2} \cdot\left(f /\left(2 \alpha_{1}\right)\right) \cdot\left(c_{p} / U_{10}\right)^{0.35}
$$

Both expressions of $H_{c r, b}^{i p}$ and $H_{c r, b}^{d m}$, characterizing the limiting amplitude of rogue waves, have been used in this study.

\section{BREAKING CRITERIA. A REVISIT}

The investigations of $\mathrm{Wu}$ and $\mathrm{Yao}$ [5] and $\mathrm{Wu}$ and Nepf [26] have added new insights, with respect to the breaking criteria that Phillips [13] and Longuet-Higgins [27] had suggested much earlier for spilling breaking and other types of wave breaking, in the presence or absence of swell. Among the three categories of breaking criteria used, namely the kinematic, dynamic, and the slope (or geometric) criterion, it appears that the kinematic one is the most fundamental. The dynamic criterion is also related to the maximum slope concept. It appears that the slope breaking criterion is related to other limiting (or maximum) average slope concepts (as described in IP). According to older field observations [28], the average slope of a wave field, locally, cannot exceed the upper limit of about 0.28 , that is:

$(\mathrm{kh})_{\mathrm{av}}=2 \sqrt{2} \pi \S \mathrm{H}_{\mathrm{av}}=8.886 \S \mathrm{H}_{\mathrm{av}}(\theta) \leq 0.28$

although some other authors (e.g., Hsu et al. [29]) have argued that in non-linear bound wave fields this threshold may reach higher values, up to about 0.32 . In summary, the breaking criteria, modified by the surface drift current effects and the non-linearity of the wave field expressed by $f$, are as follows:

Kinematic: $\gamma c \leq u_{o r b}^{c}+q_{c}$ 


$$
\text { Dynamic: } \begin{aligned}
S_{\mathrm{p}} & >S_{\max , p}\left\{=a_{L} / g=f /\left(2 \alpha_{1}\right) \text { or } S_{\max , p}\right. \\
& \left.=\left(f / 2 \alpha_{1}\right)\left[1-\alpha_{o}\left(c_{p} / u_{*}\right)^{-1}\right]^{2}\right\}
\end{aligned}
$$

Average slope: $S_{a v}=2 \sqrt{2} \pi \S \mathrm{H}_{\mathrm{av}}(\theta) \leq 0.28$

Here, $\mathrm{q}_{\mathrm{c}}$ is the value of surface drift at the wave crest, and the coefficient $\alpha_{o}$ expresses the ratio of the Eulerian surface drift (at the zero crossing point of the waveform with the mean water level) and $\mathrm{u}_{*}$, being of $\mathrm{O}(0.5)$. The constant $\gamma$ may take either of the following values: $1,0.5,0.75$, or 1.5 depending on the type of breaking examined (e.g., spilling, plunging, etc.), and on whether the orbital velocity (at the wave crest), $u_{o r b}^{c}$, on the RHS of the first inequality (18) is compared with the phase velocity, $\mathrm{c}$, or with the group velocity, $\mathrm{C}_{\mathrm{g}}$. Phillips [13] and Longuet-Higgins [27] have suggested $\gamma=1$, for spilling breakers, whereas $\mathrm{Wu}$ and Yao [5] have suggested $\gamma=0.5$ (as $\left.C_{g}=0.5 c\right)$. On the other hand, $\mathrm{Wu}$ and Nepf [26] have suggested $\gamma=1.5$ for plunging breakers. The kinematic condition (18), with the aid of an expression providing the distribution of drift current along the mean wave profile (see also IP), leads to the determination of a critical frequency $\omega_{\mathrm{cr}}$ above which all waves break, viz:

$$
\omega_{c r} / \omega_{p}=\gamma\left[1-\left[(1-a k)^{2}-\gamma_{1}\left(2-\gamma_{1}\right)\right]^{1 / 2}\right]^{-1}
$$

where $\gamma_{1} \approx 0.03\left(U_{10} / c_{p}\right)$ or $\approx \alpha_{o}\left(c_{p} / u_{*}\right)^{-1}$. For $\omega_{\mathrm{cr}}=\omega_{\mathrm{p}}$, breaking of the dominant wave occurs and:

$$
1-\left[(1-a k)^{2}-\gamma_{1}\left(2-\gamma_{1}\right)\right]^{1 / 2}=\gamma
$$

Thus, in our formulation, $\gamma \leq 1$. In other words, for plunging breakers, $u_{o r b}^{c}$ must refer to the group velocity. Therefore, $\gamma$ should (then) have the value $0.5 \times 1.5=0.75$.

As mentioned before, the concept of limiting average slope leads to the notion of premature wave breaking, or stated differently, for any given sea state (characterized by $\S$, and/or $\mathrm{U}_{10} / \mathrm{c}_{\mathrm{p}}$ ), a new local maximum slope:

$\S_{\max }^{\theta}\left[=0.28 /\left\{2 \sqrt{2} \pi \S H_{a v}(\theta)\right\}=0.0315 H_{a v}^{-1}(\theta)\right]$

can be obtained which, if exceeded, will cause the wave field to prematurely break, even if $\S$ (the slope characterizing the original sea state) is less than $\S_{\max }=0.0356 ; \S_{\max }^{\theta}$ cannot (also) exceed the corresponding Stokes limit $\S_{\max }^{\mathrm{s}}=0.0505$. $H_{a v}$ is calculated (in the form of function $\mathrm{F}_{1}(\theta)$, as described in IP) from knowledge of the wave spectrum. In this study, the spectral form proposed by Donelan et al. [30], and slightly modified by Banner [31], is used to estimate $\mathrm{m}_{\mathrm{o}}, \mathrm{m}_{2}$ and $\mathrm{m}_{4}$, and hence $\theta$, for selected values of either $\S$ and/or $\mathrm{U}_{10} / \mathrm{c}_{\mathrm{p}}$, although other forms of the sea spectrum could also be used for these calculations; $\S$ and/or $\mathrm{U}_{10} / \mathrm{c}_{\mathrm{p}}$ appear to be important parameters that characterize a given sea state. In the absence of swell, it is also possible to select only the value of $\mathrm{U}_{10} / \mathrm{c}_{\mathrm{p}}$ (perhaps, in the range proposed by Donelan et al. [30]) and then compute $\S$ from a chosen $\S-\mathrm{U}_{10} / \mathrm{c}_{\mathrm{p}}$, as that described above.

For a selected $\mathrm{U}_{10} / \mathrm{c}_{\mathrm{p}}$ value, $\S$ and the slope $B_{e f}=4 \pi \S$ are estimated, $\gamma_{1}$ is examined whether it remains less or greater than the quantity: $1-\left[B_{e f}\left(2-B_{e f}\right)\right]^{1 / 2}$ and proper values of both $\alpha_{1}\left\{=(f / 2)\left(\mathrm{a}_{\mathrm{L}} / \mathrm{g}\right)^{-1}\right\}$ and of $(\mathrm{ak})_{\mathrm{cr}}\left\{=B_{e f, c r} / \mathrm{c}_{\mathrm{p}}\right\}$ are determined as functions of $f, \gamma$ and $\gamma_{1}$; (ak) cr expresses the maximum slope that a wave field can sustain for a given sea state. More specifically, if:

A.

$$
\begin{aligned}
& \text { A. } \gamma_{1}<1-\left[B_{e f}\left(2-B_{e f}\right)\right]^{1 / 2} ; \\
& a_{L} / g=1-\left\{(1-y)^{2}+y_{1}\left(2-y_{1}\right)\right\}^{1 / 2} \\
& \alpha_{1}=(f / 2)\left[\left\{(1-\gamma)^{2}+\gamma_{1}\left(2-\gamma_{1}\right)\right\}^{1 / 2}\right]^{-1} ; \\
& (\mathrm{ak})_{\mathrm{cr}}=1-\left\{(1-\gamma)^{2}+\gamma_{1}\left(2-\gamma_{1}\right)\right\}^{1 / 2}(24 \mathrm{a}, \mathrm{b}, \mathrm{c}, \mathrm{d})
\end{aligned}
$$

Valid for: $\gamma_{1} \leq 2$

$$
\begin{aligned}
& \text { B. } \gamma_{1}>1-\left[B_{e f}\left(2-B_{e f}\right)\right]^{1 / 2} ; \\
& a_{L} / g=\left[2+\gamma-\gamma_{1}-\left[\left(2+\gamma-\gamma_{1}\right)^{2}-2\left(1+\gamma-\gamma_{1}\right)^{2}\right]\right]^{1 / 2} \\
& \alpha_{1}=(f / 2)\left[\left[2+\gamma-\gamma_{1}-\left[\left(2+\gamma-\gamma_{1}\right)^{2}-2\left(1+\gamma-\gamma_{1}\right)^{2}\right]\right]^{1 / 2}\right]^{-1} \\
& (a k)_{c r}=0.5\left[2+\gamma-\gamma_{1}-\left[\left(2+\gamma-\gamma_{1}\right)^{2}-2\left(1+\gamma-\gamma_{1}\right)^{2}\right]\right]^{1 / 2}
\end{aligned}
$$

Valid for: $\gamma_{1} \leq 2$ and $\gamma-\sqrt{2}<\gamma_{1}<\gamma+\sqrt{2}$

In both cases, $B_{e f}$ and $U_{10} / c_{p}$ should remain bounded, viz.: $\quad B_{e f} \leq 0.4432 \quad$ and $\quad U_{10} / c_{p} \leq 66.67$. For $0.17 \leq \gamma_{1} \leq 1.87$ or $5.7 \leq U_{10} / c_{p} \leq 61$, both of the inequalities: $1-\left\{\gamma_{1}\left(2-\gamma_{1}\right)\right\}^{1 / 2} \leq 0.4432$ and $\gamma-\sqrt{2}<\gamma_{1}<\gamma+\sqrt{2}$ hold (for $\gamma=0.5,0.75,1.0$ ). These relationships suggest that the local maximum steepness $(\mathrm{ak})_{\mathrm{cr}}$ of rogue waves may be a function of the spectral bandwidth $\theta$, as the latter is a function of $\S$, provided that $\S$ and $\gamma_{1}$ are interrelated. This finding is consistent with the results of $\mathrm{Wu}$ and Yao ([5], cf. their Fig. 7) which show the decreasing trend of $(\mathrm{ak})_{\mathrm{cr}}$ with increasing sea bandwidth. The results also suggest that $\alpha_{1}$ is not a universal constant but a coefficient related to the maximum wave slope concept, in agreement with the findings of Longuet-Higgins $[32,33]$. The variation of $a_{L} / g$, with 
sea state, also indicates the $a_{L}$ accelerations attained at the crest of large waves, under extreme conditions. Jenkins [34] supports the idea that these accelerations may not exceed $0.5 \mathrm{~g}$ (when the waves break), in contrast to the much larger values indicated by Fochesato et al. [35]. Yet, the former author claims that these large values represent the centripetal acceleration developed during the whirling around stage of the (plunging or projected) crest tip before it touches the forward face of the wave again (also Jenkins - personal communication). Such explanation may imply that these large accelerations (of about 5-6g), reported in the literature, somehow resemble the upward trough accelerations, which according to Longuet-Higgins $[32,33]$ calculations may become unlimited. The loop under the crest of a wave (where a large acceleration develops), may be thought of as a kind of wave trough. In shallow waters, because the breaking wave geometry is affected by wave directional spreading and focusing, it is possible that overturning rogue waves may have different properties depending on whether they are in the focusing or defocusing phase, at the breaking onset, a situation largely controlled by the maximum focusing angle and the water depth [35].

\section{PROBABILITIES OF ROGUE WAVE OCCUR- RENCE AND OF BREAKING}

These occurrence probabilities, and an average probability that characterizes the presence of rogue waves may be computed from the following relations:

$$
p\left(H>H_{c r}\right)=\int_{H_{c r}}^{\infty} p(H) d H, \quad p_{\text {orw }}\left(\Sigma_{p}\right)=\left.\int_{H_{c r}}^{\infty} p(H \mid \Sigma) d H\right|_{\Sigma=\Sigma_{p}}, \quad B_{\text {orw }, a v .}=\int_{H_{c r}}^{\infty} \int_{0}^{\infty} p(H \mid \Sigma) d \Sigma d H
$$

The breaking probability of rogue waves is estimated by:

$$
p\left(H>H_{c r, b}\right)=\int_{H_{c r, b}}^{\infty} p(H) d H
$$

Furthermore, in order to conform with the max average slope restriction, at a given $\S, \S_{\max }^{\theta}$ is calculated and examined as to whether it remains $\leq \S_{\max }^{s}$ or it exceeds the latter quantity. If the initial $\S\{=\S(\theta)\}$ remains $\leq \S_{\max }^{\theta}$, the limiting amplitude $\mathrm{H}_{\mathrm{cr}, \mathrm{b}}\left(\right.$ at $\omega_{\mathrm{p}}$ ), corresponding to the initial $\S$ and the calculated $f$ and $\alpha_{1}$ values, is estimated. Yet, if $\S(\theta)>$ $\S_{\max }^{\theta}, \S=\S_{\max }^{\theta}$ is set and a new value of $B_{e f, n}\left(=4 \pi \S_{\max }^{\theta}\right)$ is calculated and used for examining whether $B_{e f, n}$ satisfies the inequality of case $\mathrm{A}$ or case B. Using $\S_{\max }^{\theta}$, a new value of $f$ is estimated and another $\alpha_{1}$ is obtained for use with $\mathrm{H}_{\mathrm{cr}, \mathrm{b}}$, etc.

\section{RESULTS}

Figure 1 shows the distribution of exceedance probability $p\left(H>H_{c r}\right)$ against the numerical factor $\mathrm{n}$ (now in the range: $0 \leq \mathrm{n} \leq 1.5$ ), with $\theta$ as a parameter. Fig. (2) shows similar results for $p_{\text {orw }}\left(\Sigma_{p}\right)$. Fig. (3) shows the exceedance

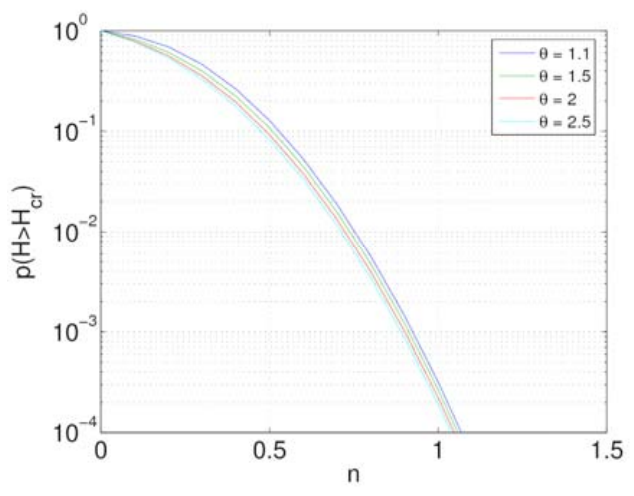

Fig. (1). Exceedance probability of crest height, using the marginal $\mathrm{p}(\mathrm{H})$ density.

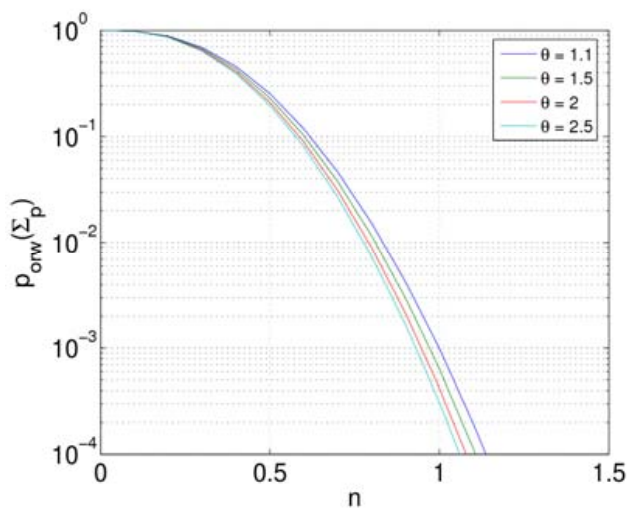

Fig. (2). Exceedance probability of crest height, using the conditional amplitude density.

probabilities $p_{n}(n)$ vs. $n$, estimated with the aid of $\mathrm{C}_{4}$, as suggested by Janssen [4]. Here:

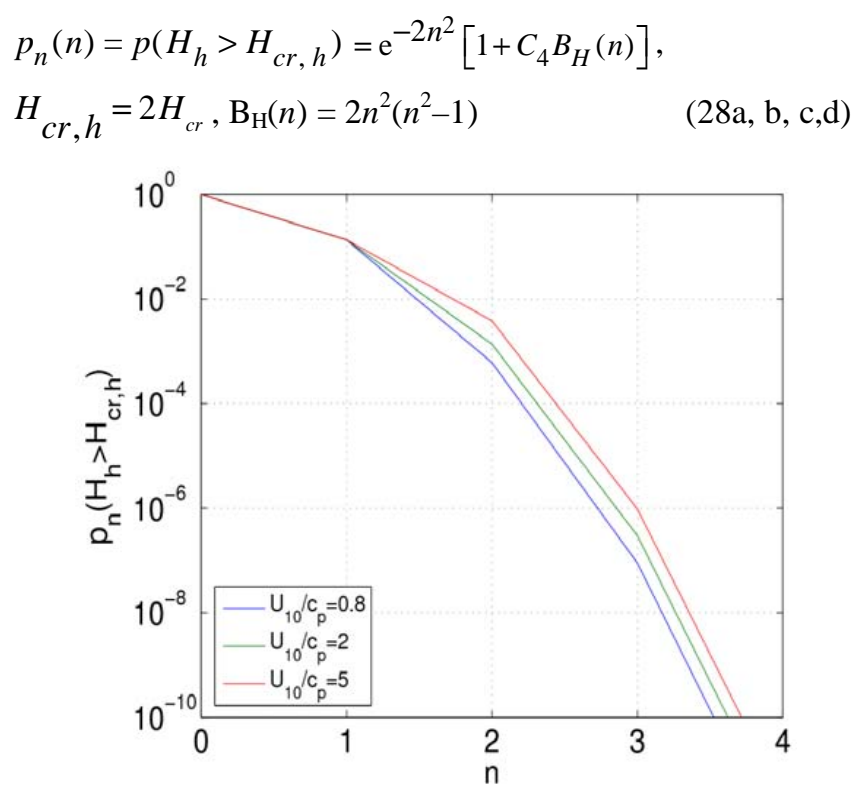

Fig. (3). Exceedance probability of wave height with the aid of $\mathrm{C}_{4}$ for various $\mathrm{U}_{10} / \mathrm{c}_{\mathrm{p}}$ values. 


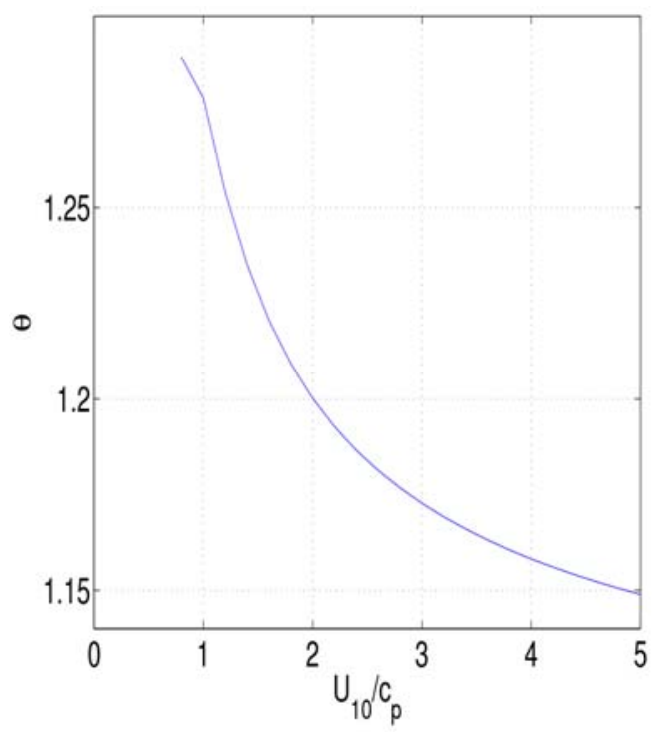

Fig. (4). Variation of $\theta$ against $U_{10} / c_{p}$.

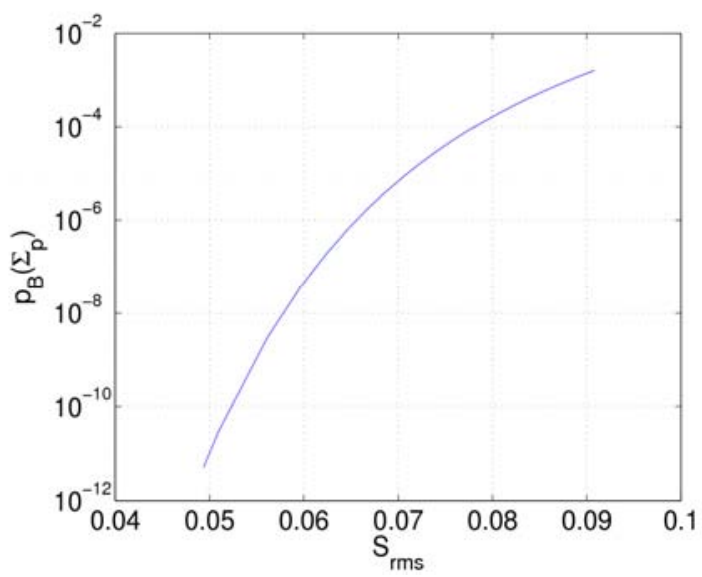

Fig. (5a). Probability of dominant wave breaking sea surface slope.

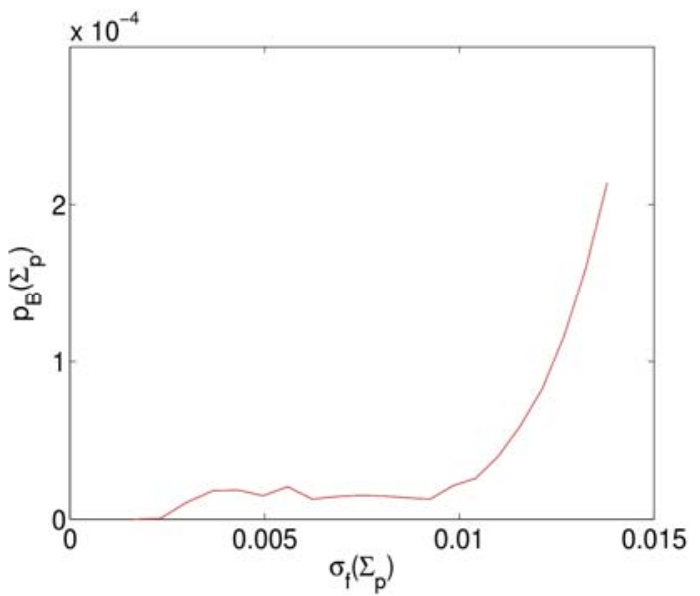

Fig. (5b). Probability of dominant wave breaking vs. the rms $S_{p}$, vs. the inverse wave age $\mathrm{U}_{10} / \mathrm{c}_{\mathrm{p}}$. by:

Note that the (normalized) limiting wave height is given

$H_{c r, h}=2 \mathrm{n} H_{s}^{n}=4 \sqrt{2} n$

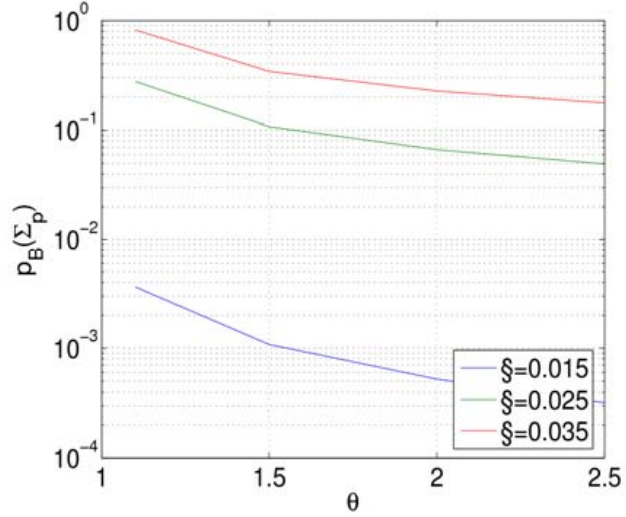

Fig. (5c). Probability of dominant wave breaking vs. sea surface bandwidth.

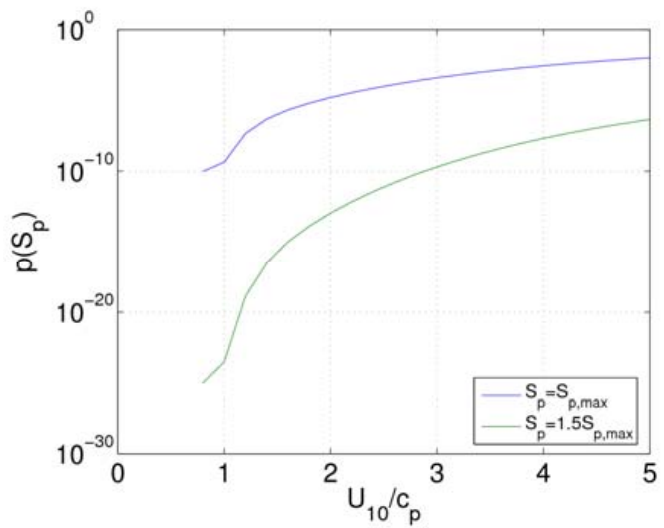

Fig. (6a). Probability distribution of peak slope, $S_{p}$, vs. the inverse wave age $\mathrm{U}_{10} / \mathrm{c}_{\mathrm{p}}$.

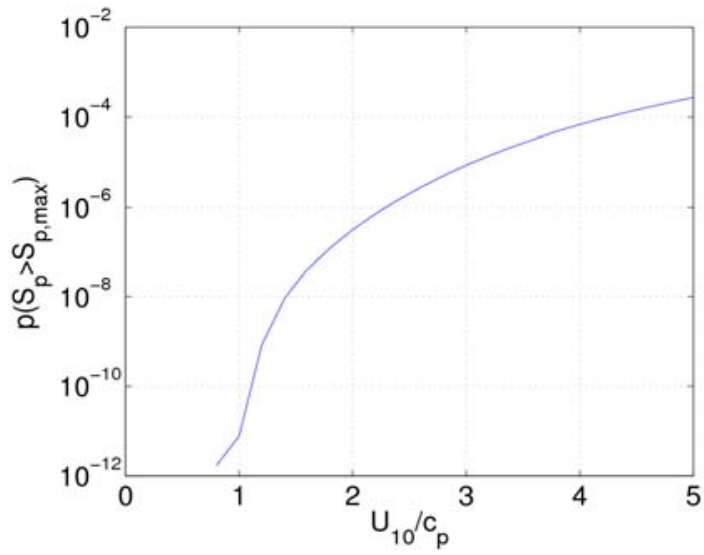

Fig. (6b). Exceedance probability of $S_{p}$, vs. $U_{10} / c_{p}$.

where now $n=0,1,2,3,4$ or 5 . On the side of this study, and for interpreting better the results obtained, the variation of $\theta$ with $\mathrm{U}_{10} / \mathrm{c}_{\mathrm{p}}$ is also shown in Fig. (4). Figs. $5(\mathbf{a}, \mathbf{b}, \mathbf{c})$ show the distributions of $\mathrm{p}_{\mathrm{B}}\left(\Sigma_{\mathrm{p}}\right)$ vs. $\mathrm{S}_{\mathrm{rms}}$, or vs. the saturation function $\sigma_{f}(\Sigma)$ or vs. $\theta$, with $\S$ as a parameter. Fig. 6(a) shows the distribution of $\mathrm{p}\left(\mathrm{S}_{\mathrm{p}}\right)$ as a function of inverse wave age, $\mathrm{U}_{10} / \mathrm{c}_{\mathrm{p}}$, whereas Figs. $\mathbf{6}(\mathbf{b}, \mathbf{c})$ show the exceedance probability $\mathrm{p}\left(\mathrm{S}_{\mathrm{p}}>\mathrm{S}_{\mathrm{p}, \mathrm{max}}\right)$ vs. $\mathrm{U}_{10} / \mathrm{c}_{\mathrm{p}}$ or vs. $\theta$, with $\S$ as a parameter. Fig. (7) shows the breaking probabilities of extreme waves against $\mathrm{U}_{10} / \mathrm{c}_{\mathrm{p}}$. Finally, Figs. 8(a, b) show the variation of $\mathrm{C}_{4}$ and BFI vs. $\mathrm{U}_{10} / \mathrm{c}_{\mathrm{p}}$, whereas Figu. (8c) shows the 


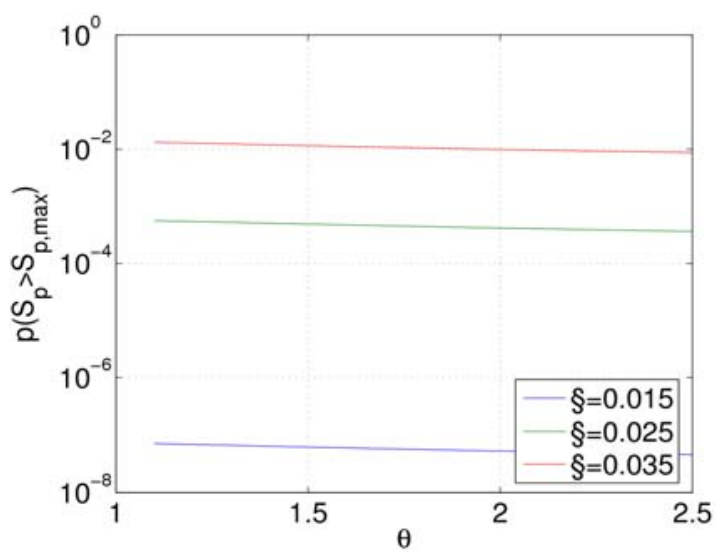

Fig. (6c). Exceedance probability of $S_{p}$ vs. $\theta$, with $\S$ as a parameter.

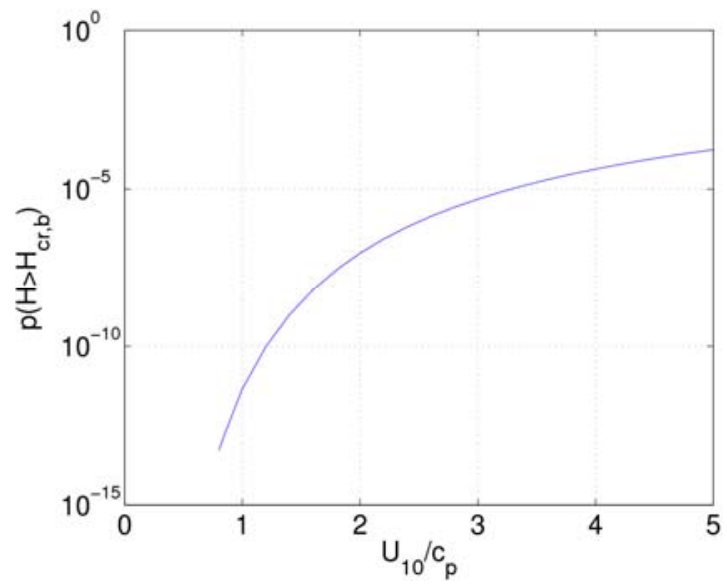

Fig. (7). Probability of rogue wave breaking vs. $U_{10} / c_{p}$.

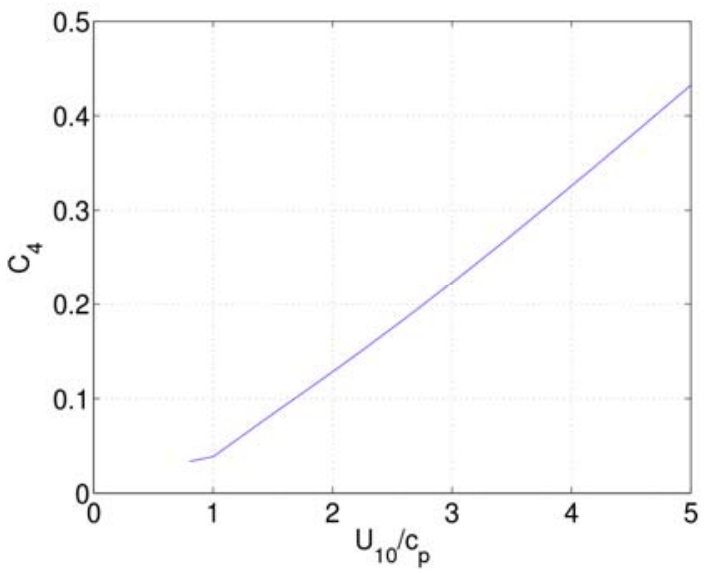

Fig. (8a). Distribution of kurtosis coefficient $\mathrm{C}_{4} \mathrm{vs}$. $\mathrm{U}_{10} / \mathrm{c}_{\mathrm{p}}$.

variation of BFI vs. $\theta ; \mathrm{C}_{4}$ is calculated from the BFI, whereas the latter index is estimated with the aid of Goda's peakedness factor, $\mathrm{Q}_{\mathrm{p}}$, that can be expressed in terms of sea spectrum, viz:

$$
\begin{gathered}
\mathrm{BFI}=2 \pi \sqrt{2 \pi} \S \mathrm{Q}_{\mathrm{p}}, \quad Q_{p}=\left(2 / m_{o}^{2}\right) \int_{0}^{\infty} \omega S^{2}(\omega) d \omega, \quad \mathrm{C}_{4}= \\
(\pi / 3 \sqrt{3}) B F I^{2} \quad(30 \mathrm{a}, \mathrm{b}, \mathrm{c})
\end{gathered}
$$

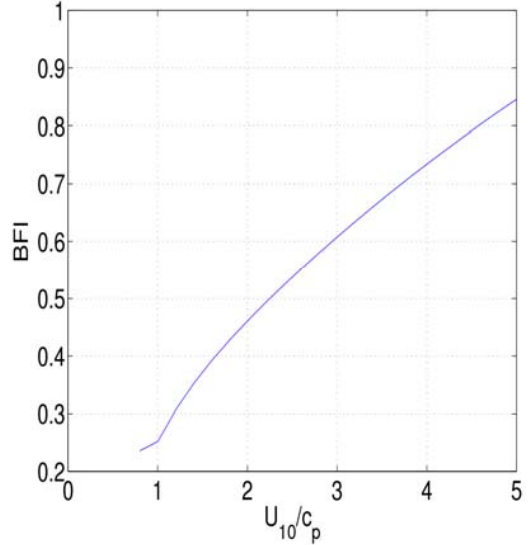

Fig. (8b). Variation of BFI against $\mathrm{U}_{10} / \mathrm{c}_{\mathrm{p}}$.

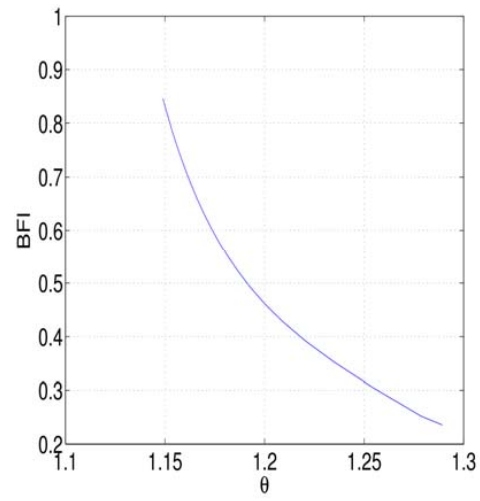

Fig. (8c). Variation of BFI against $\theta$.

\section{CONCLUDING REMARKS}

As seen from the various Figures, the statistical model of IP predicts the occurrence and breaking of extreme waves as a function of sea state, expressed in various ways (either by $\mathrm{U}_{10} / \mathrm{c}_{\mathrm{p}}$ or $\S$, or by both, and/or $\theta$, etc.). The model provides the right variation of key parameters associated with the occurrence of extreme waves, such as BFI and $\mathrm{C}_{4}$, with the sea state expressed by the above parameters. The model also yields the right variations of $\mathrm{p}_{\mathrm{B}}\left(\Sigma_{\mathrm{p}}\right)$ with both $\sigma_{f}(\Sigma)$ and other parameters $\left(S_{\mathrm{rms}}, \theta\right)$, in agreement with the Banner et al. [20] and IP results. It also provides the breaking probabilities of rogue waves.

The breaking probabilities of dominant waves and of rogue waves are estimated based on a new breaking criterion that unifies kinematic, dynamic and premature breaking concepts. With the above information available, the role of meteorological focusing in generating conditions producing rogue waves can be explored now in the Mediterranean basin, using a family of fully directionally resolved spectra wave models and reliable wind vector forcasts as well (as was done by Donelan and Magnusson [25] for the Atlantic ocean). Such work is in progress and will be reported elsewhere in the future.

\section{ACKNOWLEDGMENTS}

This research has been supported by the French Agence Nationale de la Recherche under the Project MANUREVA 
ANR-08-SYSC-019, and by the 2008 Framework Program for Research, Technological development and Innovation of the Cyprus Research Promotion Foundation under the Project $\mathrm{A} \Sigma \mathrm{TI} / 0308(\mathrm{BE}) / 05$.

\section{REFERENCES}

[1] S. R. Massel, Ocean Wave Breaking and Marine Aerosol Fluxes. Springer, 2007.

[2] K. Dysthe, H. E. Krogstad, and P. Muller, "Oceanic rogue waves", Annu. Rev. Fluid Mech., vol. 40, pp. 287-310, 2008.

[3] P. A. E. M. Janssen, "Nonlinear four-wave interactions and freak waves", In: Proc. of $14^{\text {th }}$ Aha Huliko'a Hawaian Winter Workshop, 2005, pp. 85-90.

[4] P. A. E. M. Janssen, "Nonlinear four-wave interactions and freak waves", J. Phys. Oceanogr., vol. 33, pp. 863-884, 2003.

[5] C. H. Wu, and A. Yao, "Laboratory measurements of limiting freak waves on currents", J. Geophys. Res., vol. 109, C12002, 2004.

[6] I. Papadimitrakis, "On the probability of wave breaking in deep waters", Deep-Sea Research Part II, vol. 52, pp. 1246-1269, 2005.

[7] P. Boccotti, Wave Mechanics for Ocean Engineers. Elsevier Oceanography Series 64, Elsevier, 2000.

[8] P. Boccotti, G. Barbaro, and L. Mannino, "A field experiment on the mechanics of irregular gravity waves", J. Fluid Mech., vol. 252, pp. 173-186, 1993.

[9] O. M. Phillips, D. Gu, and M. Donelan, "Expected structure of extreme waves in a Gaussian sea. Part I: Theory and SWADE buoy measurements," J. Phys. Oceanogr., vol. 23, pp. 992-1000, 1993.

[10] O. M. Phillips, D. Gu, and E. J. Walsh, "Expected structure of extreme waves in a Gaussian sea. Part II: SWADE scanning radar altimeter measurements," J. Phys. Oceanogr., vol. 23, pp. 22972309, 1993.

[11] M. A. Donelan, M. S. Longuet-Higgins, and J. S. Turner, "Whitecaps", Nature, vol. 239, pp. 449-451, 1972.

[12] J. Gemmrich, "On the occurrence of wave breaking", In: Proc. of $14^{\text {th }}$ Aha Huliko'a Hawaian Winter Workshop, 2005, pp. 123-130.

[13] O. M. Phillips, The dynamics of the upper ocean. Cambridge University Press, 1977.

[14] F. Fedele, and M. A. Tayfun, "On nonlinear wave groups and crest statistics", J. Fluid Mech., vol. 620, pp. 221-239, 2009.

[15] F. Arena, A. Ascanelli, V. Nava, D. Pavone, and A. Romolo, "Three-dimensional nonlinear random wave groups in intermediate water depth", Coastal Engineering, vol. 55, pp. 1052-1061, 2008.

[16] F. Fedele, and F. Arena, "Weakly nonlinear statistics of high random waves", Phys. Fluids, vol. 17, 026601, 2005.

[17] F. Arena, "On non-linear very large sea wave groups", Ocean Eng., vol. 32, pp. 1311-1331, 2005.

[18] T. H. Dawson, "Stokes correction for non-linearity of wave crests in heavy seas", J. Waterway, Port, Coastal Ocean Eng., ASCE, vol. 130, pp. 39-44, 2004.
[19] P. A. E. M. Janssen, "On some consequences of the canonical transformation in the Hamiltonian theory of water waves", J. Fluid Mech., vol. 637, pp. 1-44, 2009.

[20] M. L. Banner, J. R. Gemmrich, and D. Farmer, "Multiscale measurements of ocean wave breaking probability", J. Phys. Oceanogr., vol. 32, pp. 3364-3375, 2002.

[21] M. L. Banner, A. V. Babanin, and I. R. Young, "Breaking probability for dominant waves on the sea surface", J. Phys. Oceanogr., vol. 30, pp. 3145-3160, 2000.

[22] M. S. Longuet-Higgins, "Integral properties of periodic gravity waves of finite amplitude", Proc. R. Soc. London A, vol. 342, pp. 157-174, 1975.

[23] M. S. Longuet-Higgins, and M. J. H. Fox, "Theory of the almost highest wave. Part 2. Matching and analytic extension", J. Fluid Mech., vol. 85, pp. 769-786, 1978.

[24] P. Müller, C. Garrett, and A. Osborne, "Rogue waves", Oceanography, vol. 18, pp. 66-75, 2005.

[25] M. A. Donelan, and A. K. Magnusson, "The role of meteorological focusing in generating wave conditions", In: Proc. of $14^{\text {th }} \mathrm{Aha} \mathrm{Hu}$ liko'a Hawaian Winter Workshop, 2005, pp. 139-145.

[26] C. H. Wu, and H. M. Nepf, "Breaking criteria and energy losses for three-dimensional wave breaking", J. Geophys. Res., vol. 107(C10), pp. 3177, 2002.

[27] M. S. Longuet-Higgins, "On wave breaking and equilibrium spectrum of wind-generated waves." Proc. R. Soc. London A, vol. 310, pp. $151-159,1969$.

[28] B. M. Lake, and H. C. Yuen, "A new model for non-linear wind waves. Part 1. Physical model and experimental evidence". J. Fluid Mech., vol. 88, pp. 33-62, 1978 .

[29] C. T. Hsu, H. Y. Wu, E. Y. Hsu, and R. L. Street, "Momentum and energy transfer in wind generation of waves," J. Phys. Oceanogr. vol. 12, pp. 929-951, 1982.

[30] M. A. Donelan, J. Hamilton, and W. H. Hui, "Directional spectra of wind-generated waves". Phil. Trans. Royal Soc. London A, vol. 315, pp. 509-562, 1985.

[31] M. L. Banner, "Equilibrium spectra of wind waves", J. Phys. Oceanogr., vol. 20, pp. 966-984,1990.

[32] M. S. Longuet-Higgins, "Eulerian and Lagrangian aspects of surface waves", J. Fluid Mech., vol. 173, pp. 683-707, 1986.

[33] M. S. Longuet-Higgins, "Accelerations in steep gravity waves", $J$. Phys. Oceanogr., vol. 15, pp. 1590-1985, 1985.

[34] A. Jenkins, "Geometrical and kinematic properties of breaking waves in the framework of a stationary flow approximation", In: Rogue waves 2000, M. Olagnon and M. Athanassoulis (Eds.), Brest 29-30 November, 2000, pp. 221-226.

[35] C. Fochesato, S. Grilli, and F. Dias, "Numerical modelling of extreme rogue waves generated by directional energy focusing", Wave Motion, vol. 44, pp. 395-416, 2007.

Received: November 20, 2010

Revised: November 20,2010

Accepted: November 25, 2010

(C) Papadimitrakis and Dias; Licensee Bentham Open.

This is an open access article licensed under the terms of the Creative Commons Attribution Non-Commercial License (http://creativecommons.org/licenses/by-nc/3.0/) which permits unrestricted, non-commercial use, distribution and reproduction in any medium, provided the work is properly cited. 